\title{
Distribution and Genetic Diversity of Legionella pneumophila Serotype in Kuwait Environment Qadreyah Al-Matawah
}

Kuwait Institute for Scientific Research, Environment \& Life Sciences Research Center, P. O. Box 24885, Safat 13109, Kuwait

\section{Abstract}

Background: Legionella pneumophila is a waterborne organism that is increasingly recognized to cause community acquired and nosocomial pneumonia in humans. Domestic water systems have often been implicated as the source in outbreaks of Legionnaires' disease. Legionella can survive under various conditions in various water sources and acquired antibiotic resistance to many routinely prescribed antibiotics. Routine monitoring of environmental water for Legionella species is proving helpful in reducing disease outbreak.

Methods: A total of 260 of Legionella pneumophila isolates were isolated from environmental water sources from building facilities in Kuwait. The genetic diversity of the 102 isolates were analysis by the SBT method according to the European Society of Clinical Microbiology and Infectious Diseases (ESCMID) Study Group for Legionella. The distribution of Legionella isolates was investigated according to geographical region.

Results: The Legionella isolates were discriminated into 11 distinct SBT profiles, of which six (ST1223, ST1436, ST1555, ST1604, ST1718, and ST1719) were new to the ESGLI SBT database. L. pneumophila sg 7 was distributed broadly through Kuwait, accounting for $38.2 \%$ of the isolates and predominated in cooling towers water with unique sequence type to Kuwait. The second most dominant strain $L$. pneumophila sg 3 (32.4\%), predominated in the bathroom. L. pneumophila sg 1 (18.3\%), L. pneumophila sg 10 (6.9\%), and L. pneumophila sg 4 (3.9\%), predominated in the cooling towers. In SBT analysis, L. pneumophila sg 7 isolates were differentiated into 2 sequence types (STs), ST1718 (37.3 \%) is the dominant ST in cooling tower. The unique allelic profile of ST1718, obtained from the cooling tower, was not found in the ESGLI SBT database.

Conclusions: The findings of this study highlight the importance of understanding the epidemiology and ecology of L. pneumophila from public facilities in terms of public health. Furthermore, provide useful information for future epidemiological investigation of local and regional outbreaks of Legionnaires' disease.

\section{Introduction}

Legionnaires' disease (LD), usually is acquired by inhalation or aspiration of aerosol contaminated by Legionella species from environmental water sources, such as hot water supplies, cooling towers, and evaporative condensers. Potable water is considered as an important infection source in community-acquired, nosocomially acquired, and travel-associated LD cases [1-8]. Legionella spp. are gram-negative bacteria that normally occupy natural aquatic environments, where they can survive as intracellular parasites of protozoa. It includes 52 species and over 70 different serogroups. Over 20 species have been proven to be causative agents of LD. However, Legionella pneumophila species accounts for approximately $90 \%$ of confirmed cases of legionellosis. The majority of communityacquired cases are caused by strains belonging to Legionella pneumophila serogroup 1 , other non- $L$. pneumophila sg 1 strains, sg 2 to 15 , accounted for $7.4 \%$ of cases $[9,10]$. The genetic diversity of $L$. pneumophila was related to horizontal gene transfer of mobile genetic elements among L. pneumophila strains, and between different Legionella species. The potential health risk of Legionella to humans is theoretically associated with cells densities above $10^{4}$ to $10^{5} \mathrm{CFU}$ per liter of water $[11,12]$.

Commonly used method for environmental surveillance of Legionella is the standard culture technique $[13,14]$. Legionella culture is required for epidemiological typing of isolated strains to detect the source of the infection $[15,16,17]$. However, the fastidious nature of these bacteria, effect the culture technique's sensitivity to be 30 to $60 \%$ and require 3 to 7 days to grow visible colonies. Therefore, numerous typing $[16,24]$. qmutawa@kisr.edu.kw original author and source are credited.

\section{Publication History:}

Received: December 01, 2017

Accepted: February 08, 2018

Published: February 10, 2018

\section{Keywords:}

Legionaires' disease, Serogroups, SBT, ST profile, Water contamination, Cooling tower, Kuwait and applied to the epidemiological typing of L. pneumophila [18]. These methods included monoclonal antibody (MAb) subgrouping method [19] and genotyping methods, such as restriction fragment length polymorphism (RFLP) analysis [20], amplified fragment length polymorphism (AFLP) [21], and pulsed field gel electrophoresis (PFGE) [22,23]. Recently, a sequence-based typing (SBT) scheme of L. pneumophila that uses sequences from seven genes has been used in typing L. pneumophila serogroup 1 strains, and strains belonging to others serogroup [16]. The SBT method has the potential for excellent type ability, reproducibility, and epidemiologic concordance $[16,22-$ 24] and now is established as the standard sub typing technique within the European Working Group for Legionella Infections (EWGLI). The strains are assigned an SBT pattern number by EWGLI (EWGLI: www.ewgli.org) based on the sequence of the seven target genes. The method is now considered the gold standard for epidemiological

"Corresponding Author: Dr. Qadreyah Al-Matawah, Kuwait Institute for Scientific Research, Environment \& Life Sciences Research Center, P. O. Box 24885, Safat 13109 Kuwait, Tel: 0096524989116, Fax: 0096524956659; E-mail:

Citation: Al-Matawah Q (2018) Distribution and Genetic Diversity of Legionella pneumophila Serotype in Kuwait Environment. Int J Clin Med Microbiol 3: 128. doi: https://doi.org/10.15344/2456-4028/2018/128

Copyright: (C) 2018 Al-Matawah. This is an open-access article distributed under the terms of the Creative Commons Attribution License, which permits unrestricted use, distribution, and reproduction in any medium, provided the 
In Kuwait, research related to Legionella is scarce and no active surveillance program exists [25-27]. Furthermore, no reports are available on the current status of the prevalence Legionnaires' disease and associated cases in Kuwait. However, annual reports presented by the Ministry of Planning show that the death of a percentage of the population $\left(27.6 / 10^{4}\right.$ of population) is due to respiratory diseases without specifying the etiological agent [28].

Due to the following factors, it is likely that the prevalence of Legionella is underestimated; Kuwait's a hot temperate climate; the absence of water safety regulations for Legionella monitoring and decontamination; and the increased use of cooling towers within recreational and health care facilities may increase the risk of legionellosis. In addition, water temperatures in water tanks during the summer in residential compounds may be favorable for Legionella multiplication $\left(50^{\circ} \mathrm{C}\right)$. Owing to the possibility of environmental exposure to Legionella, this is the study aimed at assessing the current distribution of Legionella species from environmental water sources from public facilities such as buildings, public baths, hospitals, throughout Kuwait. Furthermore, the molecular typing of $L$. pneumophila sg isolates was conducted using sequence-based typing (SBT) to assess the genetic diversity among the isolates.

\section{Material and Methods}

\section{Legionella pneumophila}

A total number of 102 environmental isolates of L. pneumophila were used in this study. These isolates were previously isolated from domestic water system samples from different residential sites [29] and from 38 cooling towers in 12 sites in Kuwait [30]. L. pneumophila was isolated on buffered charcoal yeast extract medium using standard methods AS/NZS 3896 [31].

\section{Sero and subgrouping of Legionella pneumophila}

The Oxoid Legionella Latex test was used to identify and differentiate between sero group 1 and serogroups 2-14 (code DR0800; Oxoid; UK). The isolates were sero grouped and sub grouped, when applicable using the Dresden panel of monoclonal antibodies aspreviously described $[9,10]$.

\section{Sequence based typing (SBT)}

The genomic DNA was extracted from the isolates using the QIAamp DNA Mini kit (Qiagen). L. pneumophila isolates were genotyped using the seven gene protocol sequence-based typing (SBT) scheme developed by ESGLI as previously described [16,24]. Trace files with the obtained sequences were analyzed by using the Legionella SBT quality tool. New alleles and STs encountered for the first time in this study were submitted to the database.

\section{Whole genome sequencing (WGS)}

The genomic DNA was extracted from four representatives isolates using the QIAamp DNA Mini kit (Qiagen) and was subjected to whole genome sequencing using the Illumina MiSeq platform with $250 \mathrm{bp}$ paired-end reads according to the manufacturer's instructions. The flaA genes were extracted from the de novo assemblies (CLCbio vers. 8.0). The four isolates were selected among isolates that failed to give aflaA PCR product and showed one of the following four allelic profiles(1) F,14,16,16,15,13,2; (2) F,14,16,25,7,13,24; (3) F,14,16,65,7,13,217; or (4) F,14,16,19,15,13,215.

\section{Results}

\section{Serological distribution of Legionella species}

A total of 271 isolates of Legionella were isolated from hospital cooling tower (49\%), public buildings cooling tower (24\%), public bath $(14 \%)$, kitchen (8\%), tanks (3\%) and swimming pool (1.5\%). Seventy three percent of the total samples were collected from cooling tower water, and the rest of the samples were collected from hot water. Among the 271 isolates, the 260 L. pneumophila isolates statistically predominated (96\%), whereas Legionella species other than L. pneumophila accounted for $4 \%$ of the total. Among the 102 sequenced L. pneumophila, the sg 7 strain accounted for 39 (38\%), whereas strains sg 3 , sg 1 , sg 10, and sg 4 accounted for $32 \%, 19 \%$, $7 \%$, and $4 \%$, respectively (Figure 1 ).

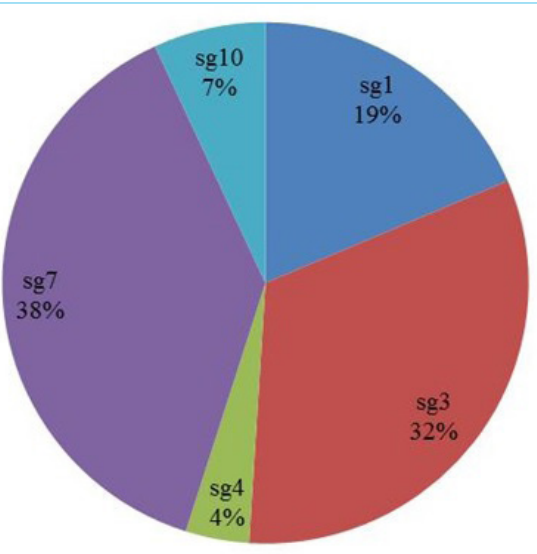

Figure 1: Distribution of Legionella pneumophila serotypes from environmental water sources in Kuwait for 2013.

\section{Analysis of geographic distribution of Legionella pneumophila}

L. pneumophila sg 1 was prevalent in 4 governorates throughout Kuwait, and was represented in $\mathrm{Al}$ Asimah (10\%), Mubarak Al-Kabeer (7.7\%), Al Ahmadi (1.3\%) and Al Farwaniyah (2.5\%). L. pneumophila sg 3 and sg 10 were represented only in Al Asimah governorate (36.3\%) and (6.3\%), respectively. L. pneumophila sg 7 was detected in 3 governorates, $\mathrm{Al} \mathrm{Asimah}(17.5 \%)$, Hawalli (5\%), $\mathrm{Al} \mathrm{Jahra} \mathrm{(5 \% ).} \mathrm{L.}$ pneumophila sg 4 was detected in 2 governorates, Al Asimah (3.8\%) and Al Ahmadi (1.3\%)(Figure 2).

\section{Legionella pneumophila serotype according to facility type}

L. pneumophila sg 7 accounted for $38.2 \%$ (39/102) of the total isolates and predominated in facilities such as buildings and cooling towers (hospitals), although not in public baths. The distribution rates of L. pneumophila strains other than sg 7 depended on the facility types; L. pneumophila sg 3 (32.4\%) prevailed in public baths, buildings and cooling towers (hospitals), L. pneumophila sg 1 (18.6\%) in public baths, buildings and cooling towers (hospitals), and L. pneumophila sg 4 in buildings and cooling towers (hospitals)(3.9\%). L. pneumophila sg 10 (6.9\%) predominated in in public baths and buildings (Figure 3 ).

\section{Legionella pneumophila sero type in cooling tower water and hot} water

In order to determine whether the distribution of Legionella pneumophila depended on the sample type, the species and sero group distributions were compared between the 72 isolates from the cooling tower and the 30 isolates from hot water (Figure 4). The predominant 


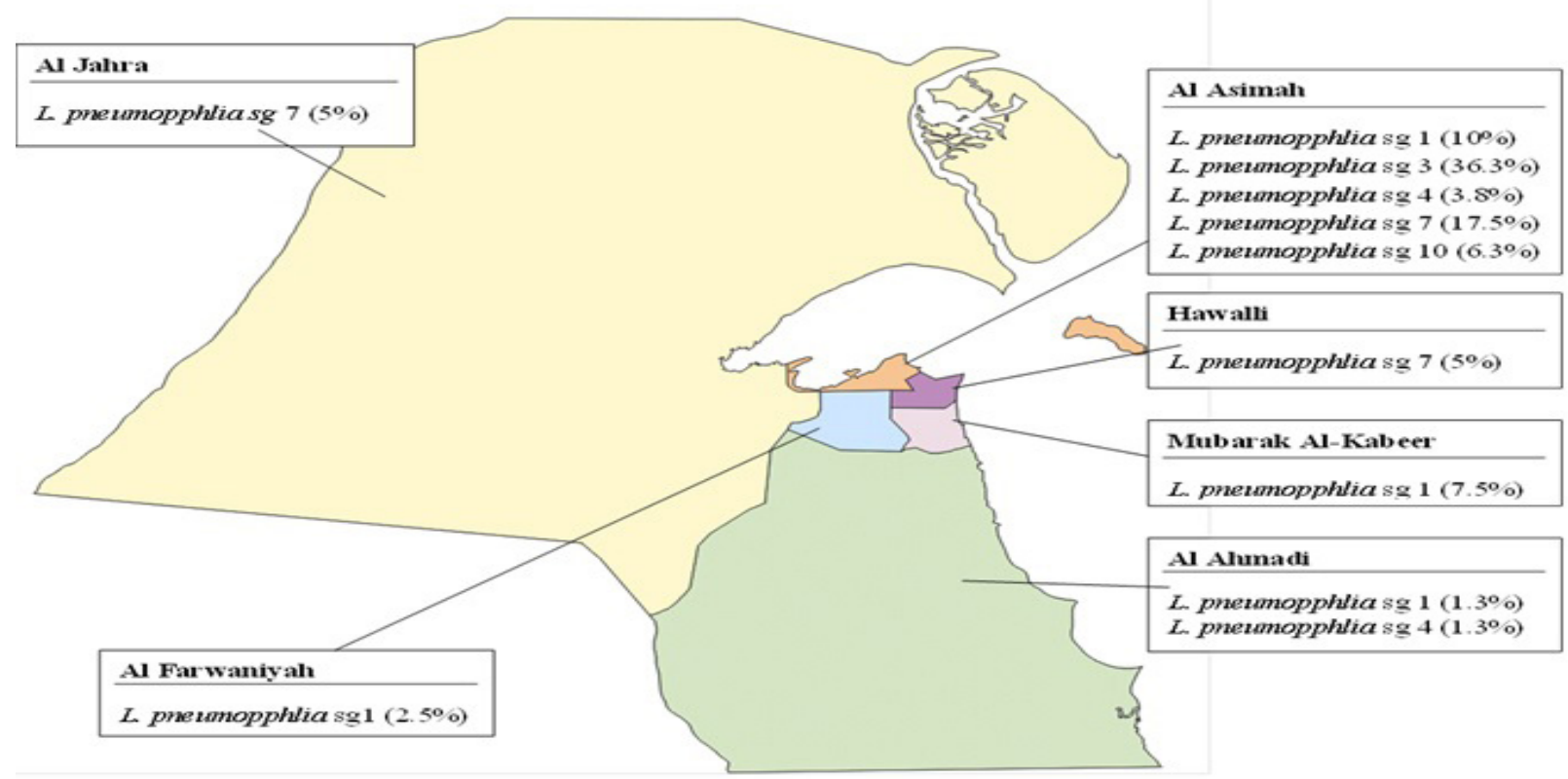

Figure 2: Distribution of Legionella pneumophila isolated from environmental water sources from 6 geographic regions in Kuwait.

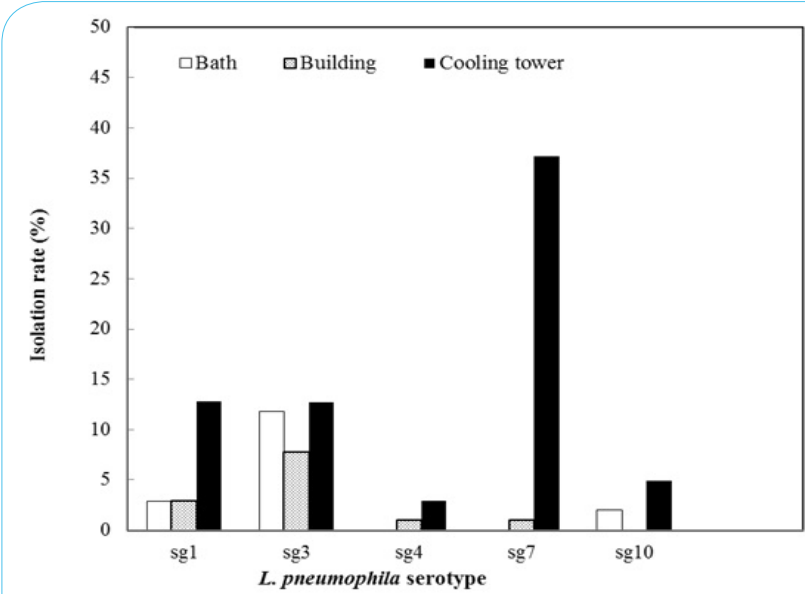

Figure 3: Comparison of Isolation rates of L. pneumophila sero type from public bath, building and cooling tower water.

strain in the cooling tower was L. pneumophila sg 7 accounted for $52.8 \%$ of isolates collected from cooling towers. The secondarily dominant strains depended on the sample type was L. pneumophila sg 3 accounted for $66.7 \%$ of isolates collected from hot water. In our comparative analysis of distribution between the cooling tower and the hot water, L. pneumophila sg 1 (18\% and $20 \%$, respectively), sg 4 (4.2\% and $3.3 \%$, respectively) and sg 10 (7\% and $6.7 \%$, respectively) were the predominant strain in both sample types.

\section{Genetic diversity}

For SBT, among 260 isolates of L. pneumophila, 102 isolates were selected randomly, and these isolates were differentiated by SBT into 11 different sequence types (STs). ST1718 of L. pneumophila sg 7, as the predominant type, accounted for $37.3 \%$, and ST1555 (1\%) was found only in one of the cooling tower samples. The profile for the two STs could not be found in the EWGLI SBT database. For L. pneumophila sg 4 (los Angeles), ST1719 (1\%) was found only in one sample of hot water, whereas ST1436 (2.9\%) of L. pneumophila sg 4 (Portland)

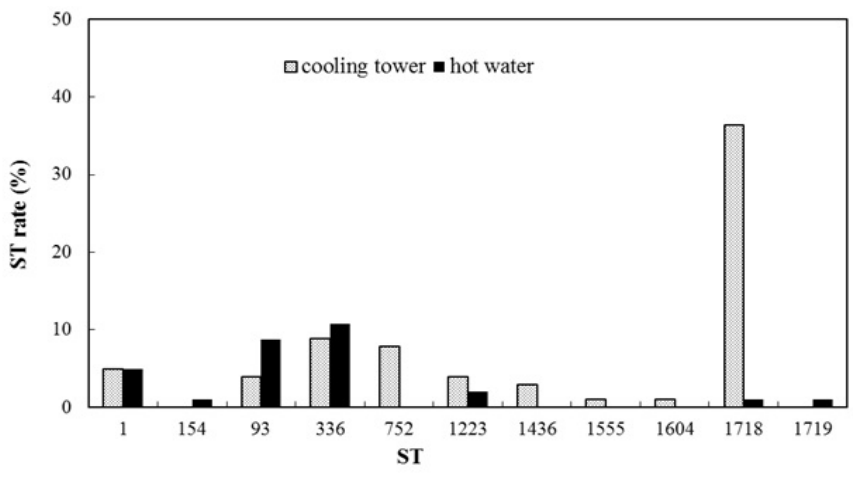

Figure 4: Comparison of ST profiles of Isolates from cooling tower water $(n=72)$ and hot water $(n=30)$.

was found only in the cooling tower. Both ST profiles could not be found in the EWGLI SBT database. ST154 (1\%) of L. pneumophila sg 1 (Oxford/OLDA) was found only in one of the hot water samples, ST $752(7.8 \%)$ only found in cooling tower samples and ST1 (9.8\%) was commonly distributed. ST1604 (1\%) of L. pneumophila sg 10 was found only in one of the cooling tower samples, ST 1223 (5.9\%) was commonly distributed. Both ST profiles could not be found in the EWGLI SBT database. ST93 (12.7\%) and ST336 (19.3\%) of $L$. pneumophila sg 3 were commonly distributed (Table 1 ).

\section{Discussion}

This study, part of it has been published, is the first study that provides the distribution and genetic diversity of environmental $L$. pneumophila serotype isolated from water in cooling towers and hot water systems in Kuwait. Among the cooling tower systems and hot water systems, the predominant species, L. pneumophila, accounted for $96.4 \%$ and $94.6 \%$ of the total isolates, respectively. L. pneumophila have been demonstrated by other studies to be the predominant 
Citation: Al-Matawah Q (2018) Distribution and Genetic Diversity of Legionella pneumophila Serotype in Kuwait Environment. Int J Clin Med Microbiol 3: 128. doi: https://doi.org/10.15344/2456-4028/2018/128

Page 4 of 5

\begin{tabular}{|l|l|l|l|}
\hline ST & $\begin{array}{l}\text { Serogroup, } \\
\text { mAb subtype }\end{array}$ & $\begin{array}{c}\text { Alleic profile } \\
\text { (flaA, pilE, asd, mip, mompS, proA, neuA) }\end{array}$ & $\begin{array}{l}\text { No. of isolates (\%) } \\
1,(9.8)\end{array}$ \\
\hline 1 & 1, Oxford/ OLDA & $1,4,3,1,1,1,1$ & $1(1)$ \\
\hline 752 & 1, Oxford/ OLDA & $11,14,16,16,15,13,2$ & $8(7.8)$ \\
\hline 93 & 1, Oxford/ OLDA & $22,4,3,1,1,1,1$ & $13(12.7)$ \\
\hline 336 & 3 & $3,10,1,28,14,9,13$ & $20(19.6)$ \\
\hline $1719^{*}$ & 3 & $11,14,16,25,7,13,24$ & $1(1)$ \\
\hline $1436^{*}$ & 4, Los Angeles & $11,14,16,65,7,13,217$ & $3(2.9)$ \\
\hline $1718^{*}$ & 4, Portland & $6,10,19,67,19,4,48$ & $38(37.3)$ \\
\hline $1555^{*}$ & 7 & $11,14,16,19,15,13,215$ & $1(1)$ \\
\hline $1223^{*}$ & 7 & $16,21,12,19,82,21,215$ & $6(5.9)$ \\
\hline $1604^{*}$ & 10 & $1,4,3,5,1,1,213$ & $1(1)$ \\
\hline
\end{tabular}

Table 1: Distribution of serogrop from 11 SBT profile for L. pneumophlia isolates ( $\mathrm{n}=102)$ in Kuwait.

${ }^{\star}$ New sequence types.

\begin{tabular}{|c|c|c|c|c|c|c|}
\hline \multirow[b]{2}{*}{ Samples Source } & \multicolumn{3}{|c|}{ No. (\%) Legionella Isolates } & \multicolumn{3}{|c|}{ No. (\%) of L.pneumophila isolates } \\
\hline & Total & L. pneumophlia & Non-L. pneumophlia & Total & $\operatorname{sg} 1$ & Non-sg1 \\
\hline Cooling tower water & 197 & $190(96.4 \%)$ & $7(3.6 \%)$ & 190 & $51(27 \%)$ & $139(73 \%)$ \\
\hline Hot water & 74 & $70(94.6 \%)$ & $4(5.4 \%)$ & 70 & $18(25.7 \%)$ & $53(75 \%)$ \\
\hline Total & 271 & $260(96.3 \%)$ & $11(4.2 \%)$ & 260 & $69(26.5 \%)$ & $192(73.6 \%)$ \\
\hline
\end{tabular}

Table 2: Comparative distribution of Legionella species between water in cooling towers and hot water $(n=271)$

species in water of cooling systems and hot water systems [3,19,32,33]. The ecology of L.pneumophila serotype was found differed between the water in cooling towers and the hot water samples collected from public facilities. L. pneumophila sg 7 was identified as a major strain $(52.8 \%)$ in water of cooling towers and L. pneumophila sg 3 as a major strain (66.7\%) in the hot water samples. L. pneumophila sg 1, compared to only $27 \%$ of the isolates collected from water in cooling towers and $25.7 \%$ of the isolates collected from hot water (Table 2). The results of this study differed from those reported in previous studies of public facilities. Specifically to $L$. pneumophila strain, sg 1 was the most frequently detected strain in cooling towers and hot water $[3,19,32,33]$.

In the comparative analysis of the SBT distribution of the isolates according to sample type, ST1718 was the predominant type in the isolates from the cooling tower water and ST336 was the dominant type in the hot water samples. However, STs 752, 1436, 1555, and 1604 were found only in isolates from the cooling tower water, and STs 154 and 1719 were found only in the hot water isolates(Figure 4). The predominant profile for sg1 in this study was ST1 $(1,4,3,1,1,1)$, which is broadly distributed throughout the world $[22,34,35,36]$. STs $1719,1436,1718,1555,1223,1604$ were not detected in the EWGLI SBT database or in any other studies. Our results are similar to those observed by Kozak et al. (2009) [37], who reported that $58 \%$ of the STs found were unique to the United States.

\section{Conclusion}

In conclusion, the results shown that the comparative populations of environmental isolates of L.pneumophila stains isolated from public facilities varied according to the types of facility as well as the geographic allocations of the facilities. Also, the results revealed several unique allelic profiles of STs and showed that ST1718 of L. pneumophila sg 7 was the prevalent sequence type in Kuwait. This study highlight the significance of understanding the epidemiology and ecology of $L$. pneumophila strain from public facilities in terms of public health and provide useful information for future epidemiological investigation of local and regional outbreaks of LD. Thus, routine monitoring of environmental water for Legionella species is an auxiliary implement to reduce the bacterial contamination of water systems and to assist the development of a more active prevention strategy. Further study will require the focus on correlation analysis by clustering between environmental and clinical isolates of Legionella strains.

\section{Competing Interests}

The author declares that he has no competing interest.

\section{Funding}

The study was supported by Kuwait Foundation for the Advancement of Sciences (KFAS) and the Kuwait Institute for Scientific Research (KISR).

\section{References}

1. Fields BS, Benson RF, Besser RE (2002) Legionella and Legionnaires' disease: 25 years of investigation. Clin Microbiol Rev 15: 506-526.

2. Joseph CA, Yadav R, Ricketts KD (2009) Travel-associated Legionnaires' disease in Europe in 2007. Euro Surveill 14: pii19196.

3. Lin $\mathrm{H}, \mathrm{Xu}$ B, Chen $\mathrm{Y}$, Wang W(2009) Legionella pollution in cooling tower water of air-conditioning systems in Shanghai, China. J Appl Microbiol106: 606-612.

4. Neil K, Berkelman R (2008) Increasing incidence of Legionella in the United States, 1990-2005: changing epidemiologic trends. Clin Infect Dis 47: 591599.

5. Ng V, Tang P, Jamieson F, Guyard C, Low DE, et al. (2009) Laboratory-based evaluation of legionellosis epidemiology in Ontario, Canada,1978 to 2006. BMC Infect Dis 9: 68-77. 
6. Palmore TN, Stock F, White M, Bordner M, Michelin A, et al. (2009) A cluster of cases of nosocomial Legionnaires disease linked to a contaminated hospital decorative waterfountain. Infect Control Hosp Epidemiol 30: 764768.

7. Ricketts KD, Joseph CA; European Working Group for Legionella Infections (2005) Legionnaires' disease in Europe 2003-2004.Euro Surveill 10: 256-259.

8. Rosmini F, Castellani-Pastoris M, Mazzotti MF, Forastiere F, Gavazzoni A, et al. (1984) Febrile illness in successive cohorts of tourists at a hotel on the Italian Adriatic coast: evidence for a persistent focus of Legionella infection. Am J Epidemiol 119: 124-134.

9. Helbig JH, Bernander S, CastellaniPastorisM, Etienne J, Gaia V et al. (2002) Pan-European study on culture-proven Legionnaires' disease: distribution of Legionella pneumophila serogroups and monoclonal subgroups. Eur J Clin Microbiol Infect Dis 21: 710-716.

10. Yu VL, Plouffe JF, Pastoris MC, Stout JE, Schousboe M, et al. (2002) Distribution of Legionella species and serogroups isolated by culture in patients with sporadic community-acquired legionellosis: an international collaborative survey. J Infect Dis 186: 127-128.

11. Best M, Yu VL, Stout J, Goetz A, Muder RR, et al. (1983) Legionellaceae in the hospital water-supply. Epidemiological link with disease and evaluation of a method for control of nosocomial legionnaires' disease and Pittsburgh pneumonia. Lancet 2: 307-310.

12. Kool JL, Bergmire-Sweat D, Butler JC, Brown EW, Peabody DJ, et al. (1999) Hospital characteristics associated with colonization of water systems by Legionella and risk of nosocomial Legionnaires' disease: a cohort study of 15 hospitals. Infect Control Hosp Epidemiol 20: 798-805.

13. Association Francaise de Normalisation. (1993) Testing water-detection and enumeration of Legionella et de Legionella pneumophila-general method by direct culture and membrane filtration. French standard AFNOR NF T90-431. Association Franc, aise de Normalisation, Paris, France.

14. International Standards Organization (1998) Water quality-detection and enumeration of Legionella. International standard ISO 11731. International Standards Organization (International Organization for Standardization) Geneva, Switzerland.

15. Fry NK, Alexiou-Daniel S, Bangsborg JM, Bernander S, Castellani- Pastoris $M$, et al. (1999) A multicenter evaluation of genotyping methods for the epidemiologic typing of Legionella pneumophilaserogroup 1: results from a pan-European study. Clin Microbiol Infect 5: 462-477.

16. Gaia V, Fry NK, Afshar B, Lück PC, Meugnier H, et al. (2005) Consensus sequence-based scheme for epidemiological typing of clinical and environmental isolates of Legionella pneumophila. J Clin Microbiol 43: 2047-2052.

17. Harrison TG, Fry NK, Afshar B, Bellamy W, Doshi N, et al. (2006) Typing of Legionella pneumophila and its role in elucidating the epidemiology of Legionnaires' disease. In: Cianciotto, N. P., Kwaik Y. A., Edelstein, P. H., Fields, B. S., Geary, D. F., Harrison, T. G., Joseph, C. A., Ratcliff, R. M., Stout, J. E., Swanson M. S. (eds) Legionella: state of the art 30 years after its recognition. ASM Press, Washington D.C., 94-99.

18. Helbig JH, Kurtz JB, Pastoris MC, Pelaz C, Lück PC, et al. (1997) Antigenic lipopolysaccharide components of Legionella pneumophila recognized by monoclonal antibodies: possibilities and limitations for division of the species into serogroups. J Clin Microbiol 35: 2841-2845.

19. Harrison TG, Doshi N, Fry NK, Joseph CA (2007) Comparison of clinical and environmental isolates of Legionella pneumophila obtained in the UK over 19 years. Clin Microbiol Infect 13:78-85.

20. Fry NK, Bangsborg JM, Bergmans A, Bernander S, Etienne J, et al. (2002) Designation of the European working group on Legionella infection (EWGLI) amplified fragment length polymorphism types of Legionella pneumophila serogroup 1 and results of intercentre proficiency testing Using a standard protocol. Eur J Clin Microbiol Infect Dis 21:722-728.

21. Drenning SD, Stout JE, Joly JR, Yu VL (2001) Unexpected similarity of pulsedfield gel electrophoresis patterns of unrelated clinical isolates of Legionella pneumophila, serogroup 1. J Infect Dis 183: 628-632.

22. Scaturro M, Losardo M, De Ponte G, Ricci ML (2005) Comparison of three molecular methods used for subtyping of Legionella pneumophila strains isolated during an epidemic of Legionellosis in Rome. J Clin Microbiol 43 : 5348-5350.

23. Gaia V, Fry NK., Harrison TG, Peduzzi R (2003) Sequence-Based Typing of Legionella pneumophila Serogroup 1 Offers the Potential for True Portability in Legionellosis Outbreak Investigation. J Clin Microbiol 41: 2932-2939.
24. Ratzow S, Gaia V, Helbig JH, Fry NK, Luck PC, et al. (2007) Addition of neuA, the gene encoding $\mathrm{N}$-Acylneuraminate cytidylyl transferase, increases the discriminatory ability of the consensus sequence-based scheme for typing Legionella pneumophila serogroup 1 strains. J Clin Microbiol 45: 1965-1968.

25. Qasem JA, Mustafa AS, Khan ZU (2008) Legionella in clinical specimens and hospital water supply facilities: molecular detection and genotyping of the isolates. Med Princ Pract 17: 49-55.

26. Al-Terkait N, Sadak A (2006) A travel abroad-associated case of Legionella pneumonia. Kuwait Med J 38: 59-60.

27. Behbehani N, Mahmood A, Mokaddas EM, Bittar Z, Jayakrishnan B, et al. (2005) Significance of atypical pathogens among community-acquired pneumonia adult patients admitted to hospital in Kuwait. Med Princ Pract. 14: 235-240.

28. Ministry of Planning (2009) Annual Statistical Abstract. Central Statistical Bureau, State of Kuwait; 46 ed.; (pp.76).

29. Al-Matawah QA, Al-Zenki SF, Qasem JA, Al-Waalan TE, BenHeji AH, et al. (2012) Detection and Quantification of Legionella pneumophila from Water Systems in Kuwait Residential Facilities. J Pathog 2012: 5.

30. Al-Matawah Q, Al-Zenki S, Al-Azmi A, Al-Waalan T, Al-Salameen F, et al. (2015) Legionella detection and subgrouping in water air-conditioning cooling tower systems in Kuwait. Environ Sci Pollut Res Int 22: 10235-10241.

31. AS/NZS 3896 (1998) Waters-examination for Legionella. Standards Australia. North Sydney NSW Australia.

32. Rivera JM, Aguilar L, Granizo JJ, Vos-Arenilla A, Gimenez MJ, et al. (2007) Isolation of Legionella species/serogroups from water cooling systems compared with potable water systems in Spanish healthcare facilities. J Hosp Infect 67: 360-366.

33. Lee HK, Shim JI, KimHE, Yu JY, Kang YH, et al (2010) Distribution of Legionella species from environmental water sources of public facilities and genetic diversity of $L$. pneumophila serogroup 1 in South Korea. Appl EnvironMicrobiol 76: 6547-6554

34. Borchardt J, Helbig JH, Lück PC (2008) Occurrence and distribution of sequence types among Legionella pneumophila strains isolated from patients in Germany: common features and differences to other regions of the world. Eur J Clin Microbiol Infect Dis 27: 29-36.

35. Chasqueira MJ, Rodrigues L, Nascimento M, Marques T (2009) Sequencebased and monoclonal antibody typing of Legionella pneumophila isolated from patients in Portugal during 1987-2008. Euro Surveill. 14: 19271.

36. Harrison TG, Afshar B, Doshi N, Fry NK, Lee JV, et al. (2009) Distribution of Legionella pneumophila serogroups, monoclonal antibody subgroups and DNA sequence types in recent clinical and environmental isolates from England and Wales (2000-2008). Eur J Clin Microbiol Infect Dis 28:781-791.

37. Kozak, NA, Benson RF, Brown E, Alexander NT, Jr. Taylor TH, et al. (2009) Distribution of lag-1 alleles and sequence-based types among Legionella pneumophila serogroup 1 clinical and environmental isolates in the United States. J Clin Microbiol 47: 2525-2535. 